\title{
Stability of Nano Encapsulated Rice Bran Derived Bioactive Pentapeptide in Apple Juice
}

Fatima Alessa ${ }^{1}$, Navam Hettiarachchy ${ }^{1 *}$, Srinivas J Rayaprolu ${ }^{1}$, Mourad Benamara ${ }^{2}$, Denise Greathouse ${ }^{3}$ and Surendra Singh ${ }^{4}$

${ }^{1}$ Department of Food Science, University of Arkansas, Fayetteville AR 72704, USA

${ }^{2}$ Department of Nanotechnology, University of Arkansas, Fayetteville AR 72701, USA

${ }^{3}$ Department of Chemistry, University of Arkansas, Fayetteville AR 72701, USA

${ }^{4}$ Physics Department, University of Arkansas, Fayetteville AR 72701, USA

\begin{abstract}
Bioactive compounds can be derived from cereal grains and their components such as rice bran. These bioactive compounds have a potency to promote health. A pentapeptide with a sequence of amino acids Glu-Gln-Arg-Pro-Arg (EQRPR) prepared from heat stablized defatted rice bran (HDRB) has demonstrated multi-site anti-cancer properties in human cell lines. Fruit juces can be used as vehicles to incorporate the pentapeptide as a nutraceutical. To address the stability of the pentapeptide in the beverage and to prevent possible interactions with other components, nano-encapsulation with poly (lactic-co-glycolic acid) (PLGA) was used to deliver the bioactive ingredient. The nano-encapsulated pentapeptide (concentrations: $200 / 400 / 600 \mu \mathrm{g} / \mathrm{mL}$ ) in the apple juice (model system) showed significant stability with no degradation for 2 months based on the high performance liquid chromatography analysis. The nanoparticles were uniform and stable with an effective diameter ranging between 82 and 83 $\mathrm{nm}$ and the results also indicated that there were no significant changes in the size over a storage period of 60 days. There was no microbial growth observed in the prepared apple juice samples. The un-encapsulated pentapeptide incorporated in the apple juice showed significant degradation after 7 days of storage. The PLGA nanoparticles showed a remarkable effect in protecting and stabilizing the bioactive compounds (pentapeptide) during the storage period at $4^{\circ} \mathrm{C}$. The PLGA nanoparticles can thus be a promising carrier for the bioactive pentapeptide when incorporated into a juice medium.
\end{abstract}

Keywords: Nano-encapsulation; Polylactic-co-glycolic acid; Pentapeptide; Nutraceuticals

\section{Introduction}

Bioactive compounds, which convey nutraceutical activity, envelop broad types of functional groups. These functional groups which exist in foods can potentially affect the biological functions. These bioactive compounds consist mainly of aromatic alcohols such as polyphenols and phenolic acids, and it has been found that these compounds have an effect in alleviating the pathology of cardiovascular disease and cancer [1]. Proteins and peptides are important bioactive compounds which can impart several physiological functions in the body, such as anti-oxidant, anti-hypertensive, anti-obesity, anti-angiogenic, hypocholestrolemic, and immune-modulatory [1]. Rice bran is a good source of dietary protein (12-20\%) and is an inexpensive co-product from rough rice-milling. A pentapeptide derived from heat stabilized defatted rice bran (HDRB) with an amino acid sequence of Glu-GlnArg-Pro-Arg (EQRPR) demonstrated anti-cancer activity, and has the potential of being incorporated in a food system [2].

Fruit juices can be good vehicles to incorporate nutraceutical s and bioactive compounds. Apple juice is consumed by $12.3 \%$ of Americans, second most popular juice after orange juice, and it also imparts health benefits due to the phenolic compounds [3]. In a previous study, orange juice was fortified with rice bran peptide fractions which were stable at $4^{\circ} \mathrm{C}$ under acidic conditions ( $\mathrm{pH} 3.0-4.5$ ) for 42 days followed by rapid degradation [4]. The results supported fruit juices as suitable vehicles to incorporate peptides and proteins; however, peptide fractions are likely to interact with other compounds in the juice matrix which affects their stability over longer storage period. The interactions among the components in fruit juices such as vitamins, minerals and phenolics can cause chemical deterioration and lead to changes in flavor and aroma of fruit juices. Nanotechnology is a fast-emerging field that provides potential benefits including enhanced stability in the controlled release and bioavailability of bioactive ingredients [5]. Nanoparticles can be defined as "a discrete entity with at least one dimension being $100 \mathrm{~nm}$ or less" [5]. In a study conducted using casein nanoparticles, hydrophobic nutraceuticals such as omega-3 fatty acid were delivered by preventing oxidation, odor, and off-flavor into beverages [6]. In another study caseinophosphopeptides (CPPs) were used to encapsulate micronutrients such as iron and added to fruit beverages such as grape, orange, and apricot puree [7]. This study concluded that the CPPs served as a good delivery system and contributed to a decrease in Fe deficiency by increasing bioavailability.

The objectives of this study were to prepare and encapsulate pentapeptide and investigate the shelf life stability of nanoencapsulated pentapeptide in apple juice at $4^{\circ} \mathrm{C}$, and to evaluate the physical attributes for 60 days.

\section{Materials and Methods}

\section{Materials}

A pentapeptide with a sequence of amino acids Glu-Gln-Arg-Pro$\operatorname{Arg}$ (EQRPR) was purchased from Biomatik USA, LLC (Wilmington, DE. Apple juice concentrate was purchased from a local grocery store. Poly (lactic-co-glycolic acid) (PLGA) was purchased from Boehringer Ingelheim Chemicals, Inc. (Ingelheim, Germany). Dimethylsulfoxide (DMSO) was purchased from Fisher Scientific (Fair Lawn, NJ) and

*Corresponding author: Navam $S$ Hettiarachchy, Department of Food Science \& Institute of Food Engineering, University of Arkansas, $2650 \mathrm{~N}$. Young Avenue, Fayetteville, AR 72704, USA, Tel: (479) 575-4779; Fax: (479) 575- 6936; Email:nhettiar@uark.edu

Received April 10, 2014; Accepted August 23, 2014; Published August 29, 2014

Citation: Alessa F, Hettiarachchy N, Rayaprolu SJ, Benamara M, Greathouse D, et al. (2014) Stability of Nano Encapsulated Rice Bran Derived Bioactive Pentapeptide in Apple Juice. J Food Process Technol 5: 356. doi:10.4172/2157-7110.1000356

Copyright: @ 2014 Alessa F, et al. This is an open-access article distributed under the terms of the Creative Commons Attribution License, which permits unrestricted use, distribution, and reproduction in any medium, provided the original author and source are credited. 
polyvinyl alcohol (PVA) was procured from Kuraray (New York, NY). The chemicals for HPLC were purchased from Sigma (St. Louis, MO), while tryptic soy agar (TSA) for total plate count (TPC) and potato dextrose agar (PDA) for mold and yeast count purchased from Becton Dickinson and Company (Spark, MD) were used to enumerate microbial survivors.

Preparation of nanoparticles using PLGA polymer and incorporation of nano-encapsulated pentapeptide in apple juice

Nano-precipitation method developed by Ravichandran et al. was used to prepare the nanoparticles [8]. The method consisted of two phases: organic and aqueous phases. The organic phase of the nanoparticles was prepared by slowly adding $0.75 \mathrm{~g}$ of PLGA $503 \mathrm{H}$ monomer into $15.0 \mathrm{~mL}$ DMSO while stirring until all the contents were dissolved. Pentapeptide at the concentrations of 200/400/600 $\mu \mathrm{g} /$ $\mathrm{mL}$ (based on an inhibitory concentration of cancer cell lines from a previous study [1]) were added to the organic phase while stirring. The aqueous phase was prepared by adding $0.05 \mathrm{~g}$ PVA into $10 \mathrm{~mL}$ of apple juice, which was prepared by mixing one part of apple juice concentrate and three parts of water to achieve the total soluble solids of $11^{\circ}$ Brix (determines the consistency and solubility of the food product), and stirred until all contents were dissolved. Three milliliters of the organic phase were taken using a syringe and slowly injected into the aqueous phase to form nanoparticles. Three types of solutions were also prepared: $1.10 .0 \mathrm{~mL}$ apple juice; $2.10 .0 \mathrm{~mL}$ of apple juice and nanoparticles; and $3.10 .0 \mathrm{~mL}$ water $(\mathrm{pH} 3.7$ to mimic $\mathrm{pH}$ of apple juice) with nanoparticles only. The samples were pasteurized at $71^{\circ} \mathrm{C}$ for $15 \mathrm{sec}$ as per FDA recommendation [9], and stored at $4^{\circ} \mathrm{C}$. The particle size and the stability of pentapeptide were tested before and after pasteurization using a particle size analyzer. The products were stored at $4^{\circ} \mathrm{C}$ for the duration of the study and evaluated at periodic intervals of $0,2,7,14,30$, and 60 days.

\section{Stability of nano-encapsulated pentapeptide in pasteurized apple juice by HPLC}

An HPLC method developed by Kannan et al. was followed to investigate the stability of the pentapeptide [2]. The samples of pentapeptide in water, pentapeptide in apple juice, nano-encapsulated pentapeptides in DI water at $\mathrm{pH} 3.7$ and nano-encapsulated pentapeptides in apple juice were analyzed by HPLC. The nanoencapsulated pentapeptides in water and apple juice with three different concentrations of pentapeptide $(200 / 400 / 600 \mu \mathrm{g} / \mathrm{mL})$, and the control of nanoparticles in water were ultra-centrifuged using ultra-centrifuge Model J2-21, Beckman Inc. (Brea, CA) at 60,000 rpm $(120,000 \mathrm{xg})$ for $30 \mathrm{~min}$ and $4^{\circ} \mathrm{C}$ to separate the nanoparticles from the solution. The supernatants were collected and filtered through 20 $\mu \mathrm{m}$ filter before the HPLC analysis. The HPLC (Hewlett Packard 1050 series, Agilent Technologies, Inc., Palo Alto, CA) equipped with a TSKGEL Super ODS column was used. The solvents used were $0.1 \%$ TFA in water and acetonitrile (50:50) and 0.1\% TFA in water, at a flow rate of $1 \mathrm{~mL} / \mathrm{min}$ with $10 \mu \mathrm{L}$ injection volume for $45 \mathrm{~min}$ and detected at $215 \mathrm{~nm}$. The following equation was used to calculate the pentapeptide concentration for the evaluation of the pentapeptide degradation over storage period: concentration $(\mu \mathrm{g} / \mathrm{mL})=($ area+47.421) $/ 1.539$ (from a standard curve of pentapeptide obtained using the HPLC method from a series of dilution of pentapeptide with the $\mathrm{pH}$ adjusted to 3.7).

Measurement of the particle size of nano-encapsulated pentapeptide in pasteurized apple juice

The particle size was measured to determine the effect of pasteurization on the shelf life stability of nano-encapsulated pentapeptide incorporated in apple juice. The mean particle diameter of nanoparticles prepared for delivering bioactives was measured by a laser particle size analyzer Model BI-9000AT Digital Correlator, Brookhaven Instruments Corporations (Holtsville, NY). In a 15.0 $\mathrm{mL}$-culture tube, $50.0 \mu \mathrm{L}$ of the nano-encapsulated pentapeptide incorporated into apple juice $(200 / 400 / 600 \mu \mathrm{g} / \mathrm{mL})$ was added to 10.0 $\mathrm{mL}$ of DI water separately. The solution was taken from the culture tube and added to the sample holder that was housed in the instrument. The measurements of particle size were recorded at 2 minute intervals.

\section{Scanning electron microscopy (SEM) of nano-encapsulated pentapeptide in pasteurized apple juice}

Scanning electron microscope, FEI Inc. (Hillsboro, OR) was used to take images of nanoparticles for morphological and chemical information which is important in determining the stability of nanoparticles [10-12]. The sample of nano-encapsulated pentapeptide in apple juice was dried by placing a drop of the sample on a gold coated double-sided tape attached on a metallic sample stand for 48 hours at room temperature before the analysis. The images of the nanoparticles in SEM were obtained using the microscope column and the sample was placed in a specimen camber under a low pressure (vacuum) conditions. Specimen position controllers were used to expose an area of beam/specimen interaction, which produced the image of nanoparticles by generating several detectable signals.

\section{Testing physical properties of pasteurized apple juice with nanoparticles contained pentapeptide}

The samples of pentapeptide in water, pentapeptide in apple juice, apple juice alone, nano-encapsulated pentapeptide in DI water $(\mathrm{pH}$ 3.7 ), and in apple juice (at $200 / 400 / 600 \mu \mathrm{g} / \mathrm{mL}$ ) were tested for color and microbiological attributes. All the measurements were conducted in triplicate using the following procedures [13].

Color: A Chroma-meter from Minolta Inc. (Osaka, Japan) was used to measure the color using a white tile to calibrate the instrument. The apple juice samples were measured in triplicate and the values for ' $L$ ', ' $a$ ', and ' $b$ ' were recorded. The ' $L$ ' value is a measure of brightness or whiteness, which ranges from 0 to 100 (if $\mathrm{L}=100$, the sample is white, and if $\mathrm{L}=0$, the sample is black). The parameter ' $\mathrm{a}$ ' is a measure of redness, which ranges from $-a$ to $+a$ (green $=-a$ and $r e d=+a)$ and ' $b$ ' is a measure of yellowness, which ranges from $-b$ to $+b$ (blue $=-b$ and yellow $=+b$ ). The differences of color were calculated using the following equations:

Chroma value $=\left(\mathrm{a}^{2}+\mathrm{b}^{2}\right)^{1 / 2}$

Hue value $=\arctan (\mathrm{a} / \mathrm{b})$

$$
\Delta E_{a b}=\sqrt{(\Delta L)^{2}}+\sqrt{(\Delta a)^{2}}+\sqrt{(\Delta b)^{2}}
$$

Where $\Delta E_{a b}$ is color different between nano-encapsulated and un-encapsulated pentapeptides.

Total soluble solids (TSS) and acidity (pH): TSS is an important parameter that defines the consistency and solubility of apple juice. A refractometer from Atago Inc. (Osaka, Japan) was used to measure total soluble solids of apple juice at ambient temperature. The recommended TSS for apple juice is $11.00{ }^{\circ} \mathrm{Brix}$. The $\mathrm{pH}$ of the solutions was determined using a pH-meter with epoxy $\mathrm{pH}$ electrode (VWR Symphony model 


\section{SB70P, VWR International, Inc., Suwanee, GA).}

Enumeration of microbial survivors: Microbiology tests were conducted to determine the effect of pasteurization. Microbial inactivation and growth during the storage period were determined using TPC and yeast and mold colony counts. Sterile peptone water $(0.1 \% \mathrm{w} / \mathrm{v})$ was used to make serial dilutions. TSA pour plates were prepared to determine the TPC and observed for colony counts after 48 hours of incubation at $30^{\circ} \mathrm{C}$ after plating. PDA pour plates were used to determine yeast and mold growth. Observations were made for microbial growth after incubating the plates at $22^{\circ} \mathrm{C}$ for 48 hours. Both the TPC and yeast and mold growth were continually observed until 60 days.

\section{Statistical analysis}

The statistical analysis was conducted using the GLM procedure of SAS 9.3 (SAS Institute, Cary, NC) and the differences among treatments for the stability, physical properties, and enumeration of the microbial survivors of the nano-encapsulated and un-encapsulated pentapeptides were determined statistically at a 5\% significance level. All values are reported as means of triplicate \pm SD.

\section{Results and Discussion}

\section{Stability of pentapeptide incorporated nanoparticles in pasteurized apple juice by HPLC}

The $\mathrm{pH}$ of DI water was adjusted to 3.7 to match that of apple juice for comparison of the stability of nano-encapsulated pentapeptide over a storage period. The percentage degradation of pentapeptide at three different concentrations (in water and apple juice) over the storage period was calculated using the equation $\left(R^{2}=0.99\right)$ derived from the standard curve plot. Pure apple juice (reconstituted from concentrate) was spiked into the HPLC column to show the absence of any peptides that have the same retention time as the pentapeptide. The apple juice containing known concentrations of pentapeptide (200/400/600 $\mu \mathrm{g} /$ $\mathrm{mL}$ ) was spiked to confirm the retention time of the pentapeptide which was between 11 and 13 minutes from the start of the run. Figure 1 is the HPLC profiles of the un-encapsulated pentapeptide in water $(\mathrm{pH} 3.7)$ showing degradation over time. Hence, the HPLC profiles for only 400 $\mu \mathrm{g} / \mathrm{mL}$ are shown. The HPLC analysis showed significant degradation ( $\mathrm{P}$ value $<0.001$ ) of un-encapsulated pentapeptide (at three different concentrations) in water from the 0 day: $(200 \mu \mathrm{g} / \mathrm{mL}-87.8 \%, 400 \mu \mathrm{g} /$ $\mathrm{mL}-97.4 \%, 600 \mu \mathrm{g} / \mathrm{mL}-91.9 \%)$ to the 60 th day: $(200 \mu \mathrm{g} / \mathrm{mL}-41.8 \%$, $400 \mu \mathrm{g} / \mathrm{mL}-60.8 \%, 600 \mu \mathrm{g} / \mathrm{mL}-55.8 \%)$ of storage. This could be due to the low $\mathrm{pH}$ (3.7) of the solution and possible interactions between the pentapeptide and water molecules which have been reported in previous studies [14].

Figure 1 also shows the HPLC profile of apple juice alone, which is composed of organic acids including quinic acid, citric acid, galacturonic acid, and malic acid, and amino acids including aspartic, asparagine and glutamic acids [15]. The retention times of the HPLC analysis of apple juice is representative of these components. The HPLC profiles in Figure 3 shows the un-encapsulated pentapeptide at three different concentrations in apple juice with significant degradation over storage period 0 day: $(200 \mu \mathrm{g} / \mathrm{mL}-96.6 \%, 400 \mu \mathrm{g} / \mathrm{mL}-98.1 \%, 600 \mu \mathrm{g} /$ $\mathrm{mL}-94.5 \%)$ to the 60 th day: $(200 \mu \mathrm{g} / \mathrm{mL}-60.6 \%, 400 \mu \mathrm{g} / \mathrm{mL}-67.5 \%, 600$ $\mu \mathrm{g} / \mathrm{mL}-59.1 \%)$. The effect of $\mathrm{pH}$ and the possible interactions with the components of apple juice (organic and amino acids) are the possible reasons for the degradation of the pentapeptide which is shown in Figure 2. The degradation rate of pentapeptide over 60 day study was similar in both water and apple juice and hence can be attributed to the low $\mathrm{pH}$.

Figure 3 show the HPLC profiles of nano-encapsulated pentapeptide incorporated water at $\mathrm{pH}$ of 3.7 and apple juice are stable through the duration of the experiment. In this case, Figure 4 shows an illustration of nanoparticles and pentapeptide attached through electrostatic interaction. The electrostatic interactions occur when the nanoparticles that have the negatively/positively charged surface attach to the peptide which has the opposite charge. In a study conducted by Khairallah, peptide fractions derived from rice bran were incorporated in orange juice to test the storage stability [4]. The peptide fractions were found to degrade significantly during the 0 to 42 day storage period. The researcher indicated that the degradation might have occurred due to interactions between peptide fractions and the orange juice components. Therefore, encapsulating the pentapeptide can considerably reduce the interactions and potentially lead to prolonged stability.

\section{Particle size of nano-encapsulated pentapeptide in pasteurized apple juice}

Particle size is the most important characteristic that should be monitored for successful encapsulation, loading, and release of pentapeptide. This can also affect nanoparticle stability and the delivery of bioactives. Particle size of pasteurized nano-encapsulated pentapeptide in water $(\mathrm{pH}$ of 3.7) at varying concentrations of pentapeptide $(200 / 400 / 600 \mu \mathrm{g} / \mathrm{mL})$ was measured from day 0 to day 60 using particle size analyzer. Figure 5 shows the particle size of the nanoparticles which ranged from 82 to $83 \mathrm{~nm}$ and remained stable during the storage period ( $\mathrm{P}$ value $<0.05$ ). The uniformity of the particle diameter indicated that the prepared nanoparticles were of even size and the pasteurization had no deleterious effect. Particle size for nanoencapsulated pentapeptide in apple juice with varying concentrations of pentapeptide $(200 / 400 / 600 \mu \mathrm{g} / \mathrm{mL})$ was measured from 0 to 60 days, and the diameter of particle size ranged from 82 to $83 \mathrm{~nm}$. The results from the particle size analysis showed successful stability of pentapeptide since they had large surface area to the volume ratio.

\section{Scanning electron microscopy (SEM) of nano-encapsulated pentapeptide in pasteurized apple juice}

Scanning electron microscopy was used to determine the form and stability of nanoparticles during the storage period. Images of the nanoencapsulated pentapeptide $(400 \mu \mathrm{g} / \mathrm{mL})$ in apple juice were taken at 0 , $2,7,14,30$, and 60 days of storage period using the SEM. Figure 6 shows the SEM images of the nano-encapsulated pentapeptide in apple juice $(400 \mu \mathrm{g} / \mathrm{mL})$ from 0 day and 60 day storage. The nanoparticles showed spheroidal shape, which was stable over the storage period. Thus, it can be concluded that the nano-encapsulation provided protection from degradation to the pentapeptide in the apple juice environment.

\section{Physical properties of nano-encapsulated pentapeptide in pasteurized apple juice}

Color: Color is an important parameter which can provide an image for the consumer about the quality and acceptance of a food product. The analysis of the apple juice color was based on the lightness (L), redness (a), and yellowness (b). Chroma is a significant parameter of color which indicates the intensity and relates to consumer appeal. Hue of a food product indicates the actual color of the material and contributes to the overall color expectation from the product. Total color difference, $\Delta \mathrm{E}$, is the magnitude of overall color difference 
Citation: Alessa F, Hettiarachchy N, Rayaprolu SJ, Benamara M, Greathouse D, et al. (2014) Stability of Nano Encapsulated Rice Bran Derived Bioactive Pentapeptide in Apple Juice. J Food Process Technol 5: 356. doi:10.4172/2157-7110.1000356

0 day

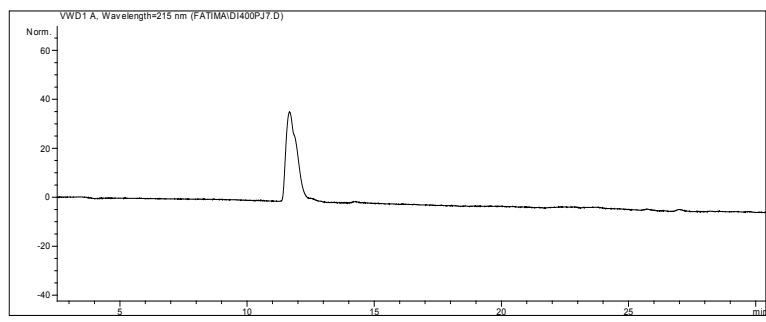

Pentapeptide incorporated in water at $\mathrm{pH}$ of 3.7
60 days

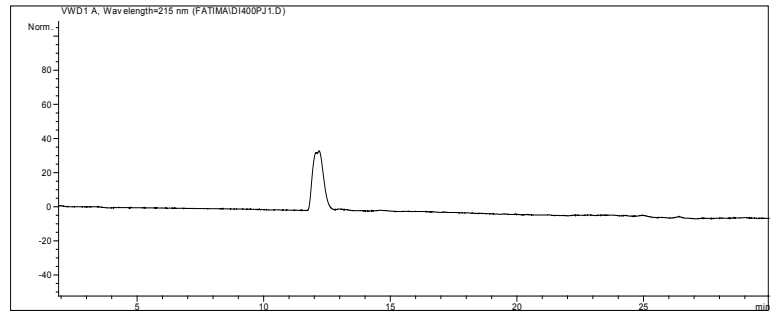

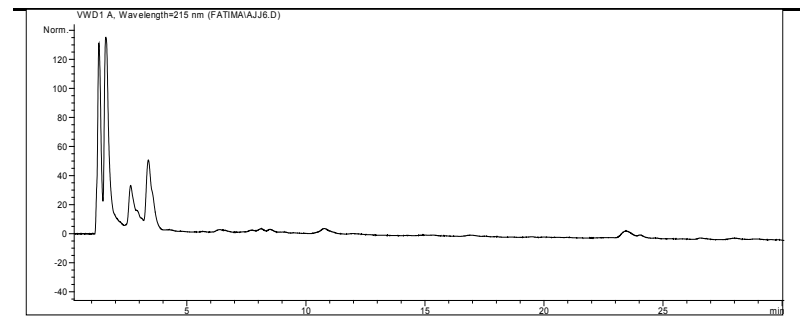

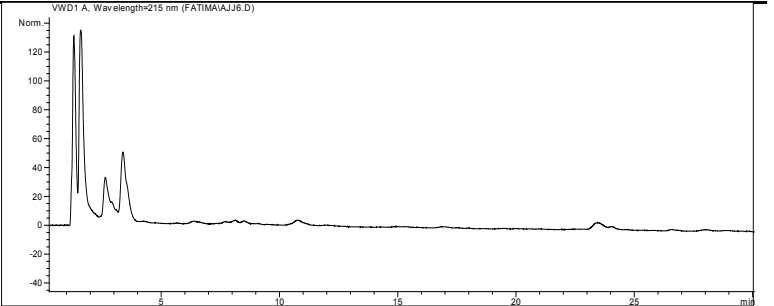

Apple juice alone
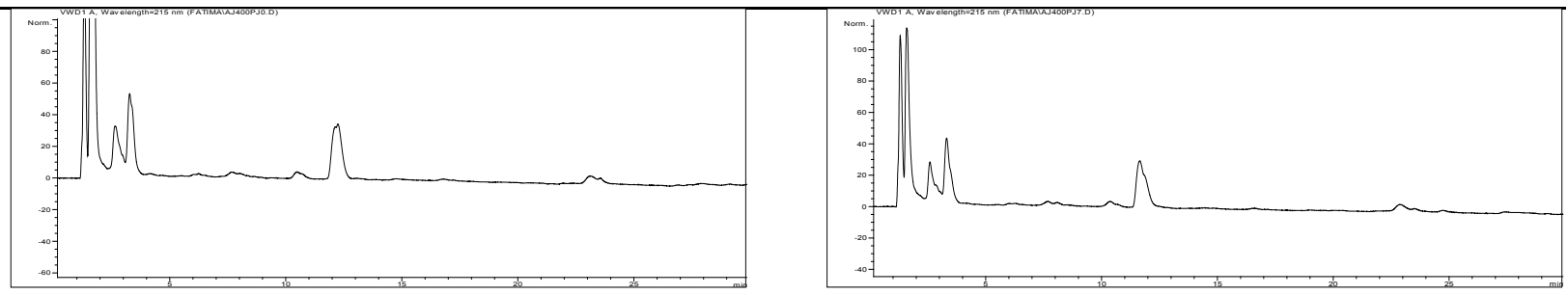

Pentapeptide incorporated in apple juice

Figure 1: HPLC profiles of pentapeptide $(400 \mu \mathrm{g} / \mathrm{mL})$ incorporated in water at $\mathrm{pH} 3.7$, apple juice alone, and pentapeptide incorporated in apple juice stored at 0 (left) and 60 (right) days. Solvent system: $0.1 \%$ TFA in water and $0.1 \%$ TFA in water and acetonitrile (50:50); flow rate: $1 \mathrm{~mL} / \mathrm{min}$; injection volume: $10 \mu \mathrm{L}$; elution time: $45 \mathrm{~min}$; absorbance measured: $215 \mathrm{~nm}$; retention time of pentapeptide: $12.0 \mathrm{~min}$.

compared between the apple juice alone (control) and the nanoencapsulated pentapeptide in apple juice [14]. Figure 7 show Chroma, Hue, and $\Delta \mathrm{E}$ for the two beverage samples over the storage period of 0 to 60 days.

Chroma of apple juice alone did not show any significant changes during the storage period. For the apple juice incorporated with varying concentrations of pentapeptide $(200 / 400 / 600 \mu \mathrm{g} / \mathrm{mL})$ Chroma measurements over the storage period (0-60 days) ranged from 0.5 to 0.3 . The results indicated that there was no significant change in the Chroma values among the three concentrations of pentapeptide in apple juice (controls). The Chroma of nano-encapsulated pentapeptide in apple juice during the storage period ranged from 3.2 to 2.8 .

The Hue angle of the apple juice alone was stable over the storage period of 60 days, and it was intensely colored (yellow). The Hue angle of apple juice containing varying concentrations of pentapeptide $(200 / 400 / 600 \mu \mathrm{g} / \mathrm{mL})$ ranged from -0.1 to 0.1 . There is no difference in the Hue among the three concentrations of pentapeptide incorporated apple juice. In a previous study investigating the stability of peptide fractions in orange juice, the changes in the color of orange juice control samples and the orange juice with rice bran peptide fractions was similar over the storage time [4]. These results are consistent with our findings, however, the addition of nanoparticles to the apple juice changed its color to light yellow and the Hue measurements were significantly different over the storage period. The results indicated that the Hue of the nano-encapsulated pentapeptide in apple juice was stable over the storage period which showed Light yellow color. The negative values for Hue angles might be due to the negative values of "a" since the Hue angle is a function of "a" and " $b$ " values.

Total color difference $(\Delta \mathrm{E})$ between the control and the sample throughout the storage period between the apple juice alone and the nano-encapsulated pentapeptide incorporated apple juice ranged from 15.2 to 14.2. The results from the color studies indicated significant differences between the apple juice alone (control) and apple juice with the nano-encapsulated pentapeptide. The difference in color remained consistent during the storage period, of 0 to 60 days which is attributed to the addition of nanoparticles which were white in color. These changes in color are significant between the treatments (apple juice and nano-encapsulated pentapeptide); while there was no significant changes over the storage time. The pasteurization temperature of 
Citation: Alessa F, Hettiarachchy N, Rayaprolu SJ, Benamara M, Greathouse D, et al. (2014) Stability of Nano Encapsulated Rice Bran Derived Bioactive Pentapeptide in Apple Juice. J Food Process Technol 5: 356. doi:10.4172/2157-7110.1000356

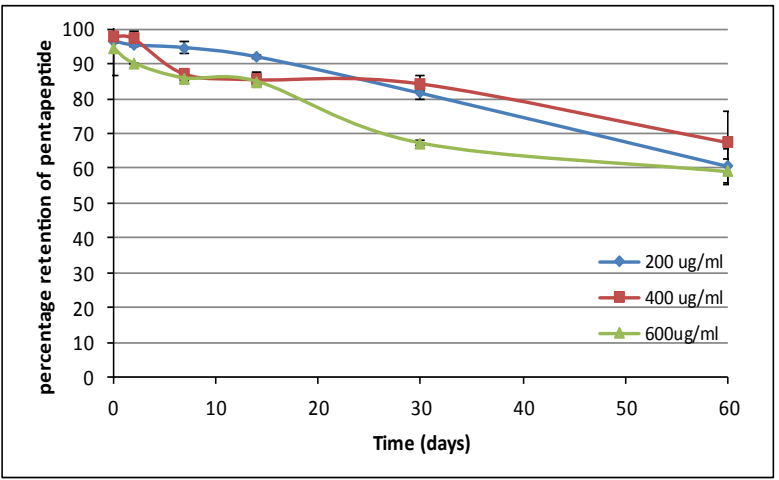

Pentapeptide in water at a $\mathrm{pH} 3.7$

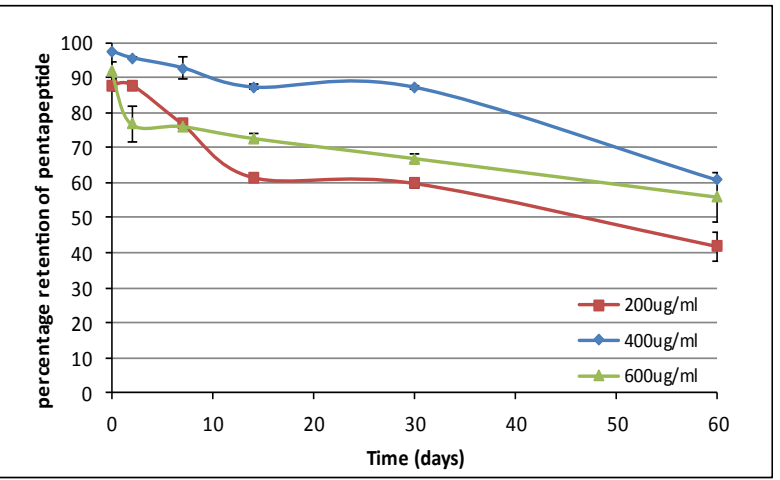

Pentapeptide in apple juice based

Figure 2: The stability of varying concentrations of pentapeptide based on the percentage degraded over a storage period $(0,2,7,14,30$, and 60 days). The percentage of degradation was calculated using the equation $\left(R^{2}=0.99\right)$ derived from standard curve plot. The values are represented as means of replicate analysis \pm standard deviation with $\mathrm{P}$ value $<0.001$

0 day

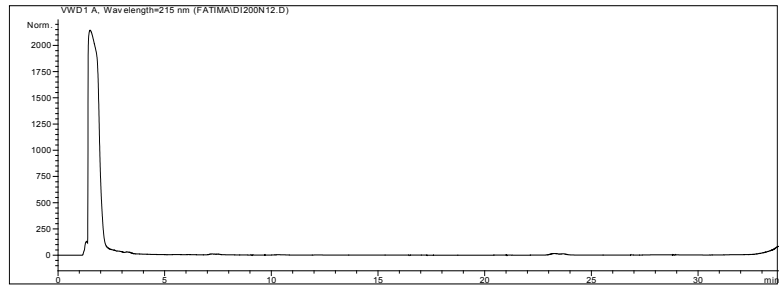

60 days

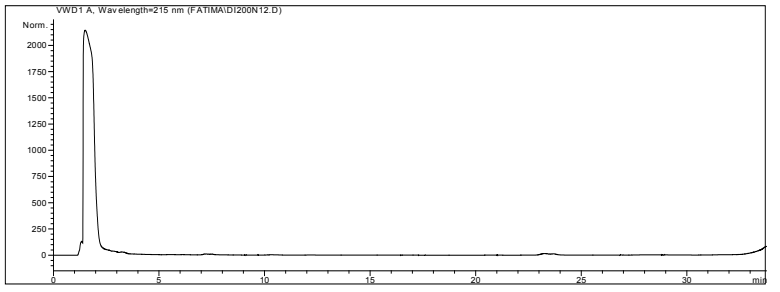

Nano-encapsulated pentapeptide $(200 \mu \mathrm{g} / \mathrm{mL})$ incorporated in water at $\mathrm{pH} 3.7$
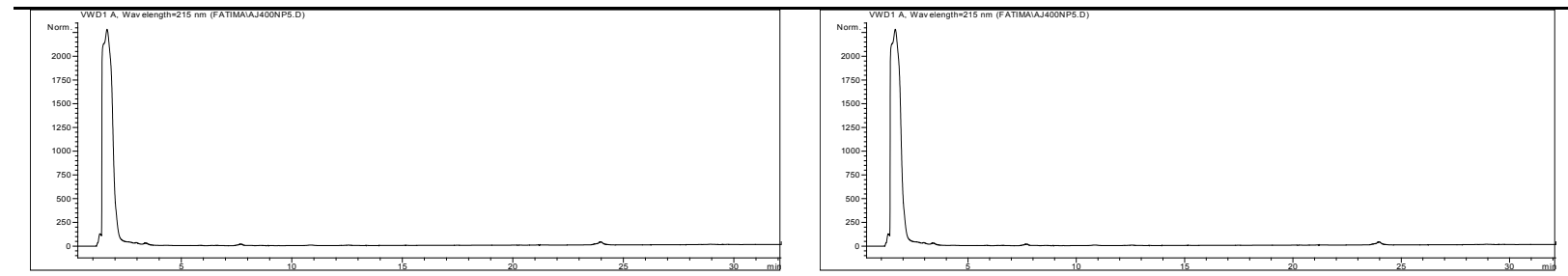

Nano-encapsulated pentapeptide $(400 \mu \mathrm{g} / \mathrm{mL})$ incorporated in apple juice

Figure 3: HPLC profiles of nano-encapsulated pentapeptide stored at 0 (left) and 60 (right) days. DMSO peak is shown on the graph (retention time 1.8 $\mathrm{min}$ ). Solvent system: $0.1 \%$ TFA in water and $0.1 \%$ TFA in water and acetonitrile (50:50); flow rate: $1 \mathrm{~mL} / \mathrm{min}$; injection volume: $10 \mu \mathrm{L}$; elution time: 45 min; absorbance measured.

$71^{\circ} \mathrm{C}$ for 30 seconds did not affect the color during the storage period. Pasteurization and low storage temperature of $4^{\circ} \mathrm{C}$ were effective in preventing significant changes or deterioration of color during storage which is consistent with a published research [16].

Acidity (pH): The $\mathrm{pH}$ of apple juice is an important factor that can determine the stability of pentapeptide. The $\mathrm{pH}$ of the nanoencapsulated pentapeptide in apple juice samples and the controls from zero to 30 days did not show any significant change. The results indicated that the $\mathrm{pH}$ remained stable for the samples at 3.7, which is within the range of apple juice $\mathrm{pH}$ (3.00 to 4.00$)$ that prevented any degradation of the nutritional components. After 60 days the $\mathrm{pH}$ of the (controls) pentapeptide in apple juice with varying concentrations $(200 / 400 / 600 \mu \mathrm{g} / \mathrm{mL})$ dropped to 3.3 , which was not significant. This change in $\mathrm{pH}$ might be due to the glutamic acid in the pentapeptide, which is negatively charged that maintained the $\mathrm{pH}$ in the acidic range [4]. In a previous study peptide fractions showed rapid degradation when incorporated into orange juice at $\mathrm{pH} 3.5$ in comparison to orange juice stored at $\mathrm{pH}$ 7.2. This was purported because of the presence of negatively charged glutamic and aspartic acid in the peptide fractions 


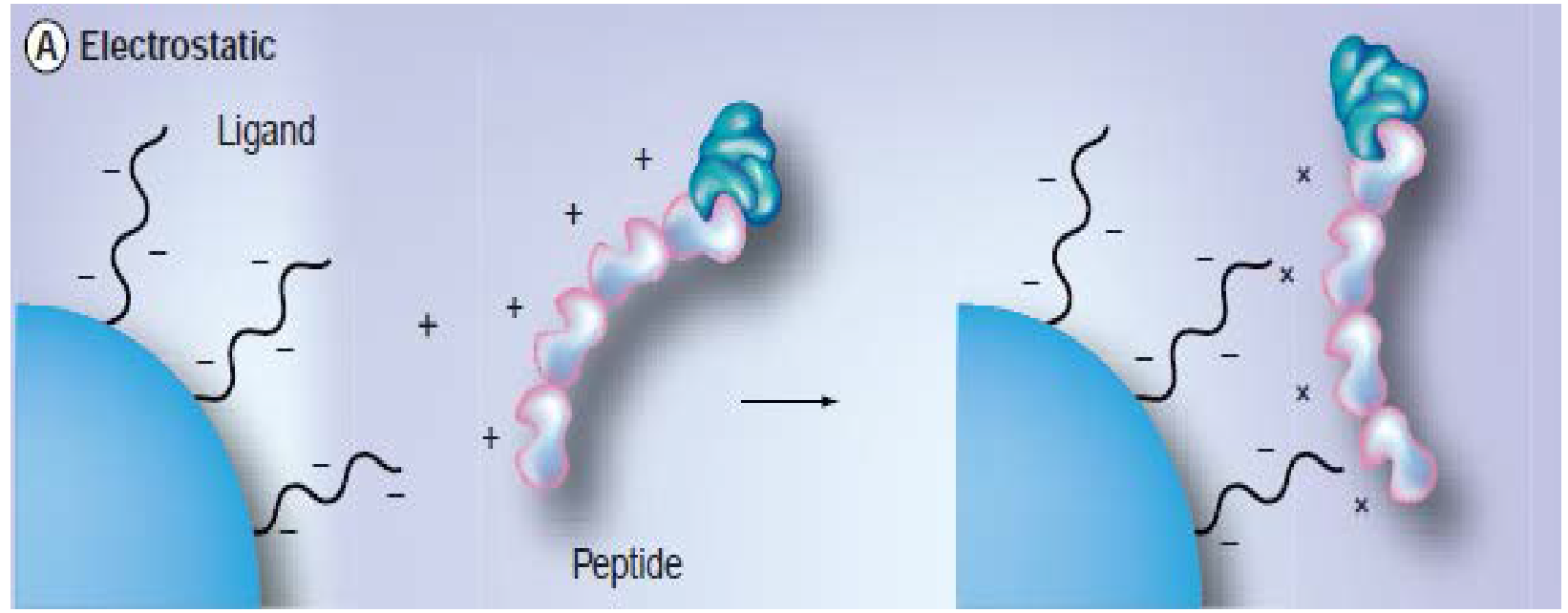

Figure 4: Illustration of electrostatic interactions between nanoparticles and peptide.

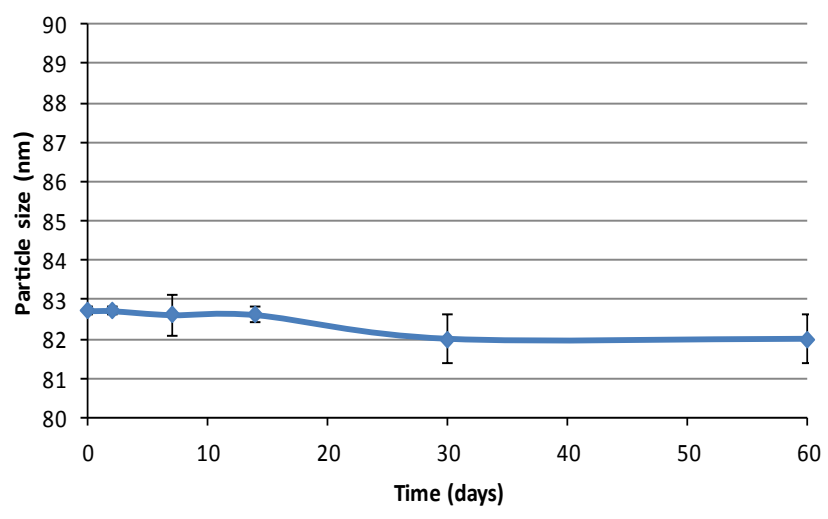

Figure 5: The particle size stability of nano-encapsulated pentapeptide in apple juice over the storage period $(0,2,7,14,30$, and 60 days). The values are represented as means of replicate analysis \pm standard deviation with $P$ value $<0.05$.

[4]. The addition of pentapeptide did not affect the $\mathrm{pH}$ of apple juice since there was no significant difference between apple juice and pentapeptide incorporated apple juice. Similar observations were recorded by previous researchers on the stability of proteins and protein hydrolysates in acidic beverages $[4,17]$.

Total soluble solid: Total soluble solid (TSS) is a significant factor to determine the consistency of color, clarity and solubility of apple juice. The TSS of apple juice was adjusted to $11^{\circ} \mathrm{Brix}$ which is consistent with that of commercially available apple juice. The total soluble solids of the control samples, apple juice alone and pentapeptide incorporated apple juice, at $0,2,7,14,30$, and 60 days were 11 and 11.3 brix respectively. The incorporation of pentapeptide in the apple juice did not significantly affect the TSS. The TSS of nano-encapsulated pentapeptide in apple juice at $0,2,7,14,30$ and 60 days was $13{ }^{\circ}$ Brix. Hence, there was a significant difference in TSS between the control samples and the nano-encapsulated pentapeptide in apple juice which was due to the addition of hydrophobic PLGA nanoparticles.

\section{Enumeration of microbial survivors}

The microbial growth was determined during the storage period using TSA plates for the TPC. The PDA was used to count the yeast and mold colonies. The TSA and PDA plates were prepared to enumerate the microbial colonies during the entire shelf life of the control samples, pentapeptide incorporated in water and apple juice, and nano-encapsulated pentapeptide in apple juice. No microbial growth was observed on both TSA and PDA plates during the storage period of 60 days, which indicated that the pasteurization of apple juice at $71^{\circ} \mathrm{C}$ was effective in inhibiting microbial growth. Furthermore, apple juice is rich in phytochemicals, which can be defined as non-nutrient plant components including polyphenols, flavonoids, hydroxycinnamic acids, dihydrochalcones, flavonols (quercetin glycosides), catechins and oligomeric procyanidins which are known for antioxidant activities [18]. These phytochemicals have also shown significant antimicrobial activity against human microorganisms [19].

\section{Conclusion}

PLGA nanoparticles are used to encapsulate the pentapeptide due to its biodegradability and compatibility. PLGA has been extensively used to deliver proteins and peptide and it is approved by FDA (20). PLGA can be degraded in vivo enzymatically or non-enzymatically producing toxicologically safe monomers [20]. Nano-precipitation method was used to prepare nano-encapsulated pentapeptide since it is a simple method of preparation and fabrication of polymeric nanoparticles such as PLGA [21]. The formation of nanoparticles by nano-precipitation does not require any external input such as high shearing, sonication, or homogenization [21]. It is a procedure with low energy cost and modest equipment, and a variety of solvents can be used such as DMSO or acetone. Furthermore, this method aids in preparing nano-dispersion in one step, decreasing energy input, and increasing encapsulation yield. Peptides provide several unique features in comparison to proteins and lipids; thus, they are more efficient for therapeutic delivery applications. Peptides can be easily sequenced and synthesized in the laboratory; therefore, they are economical. Peptides can be very specific and bind with high affinity to their cognate receptors, since they are naturally occurring from protein precursors [22]. Furthermore, multifunctional groups of peptides can be incorporated into the nanoparticles to produce a substance containing individual nanoparticle with multiple purposes [22]. Peptides are very attractive and useful molecules for the evolution of nanoparticles; however, maintaining biactivity and stability of peptides are two main challenges facing the food industry. Nano-encapsulation 
Citation: Alessa F, Hettiarachchy N, Rayaprolu SJ, Benamara M, Greathouse D, et al. (2014) Stability of Nano Encapsulated Rice Bran Derived Bioactive Pentapeptide in Apple Juice. J Food Process Technol 5: 356. doi:10.4172/2157-7110.1000356

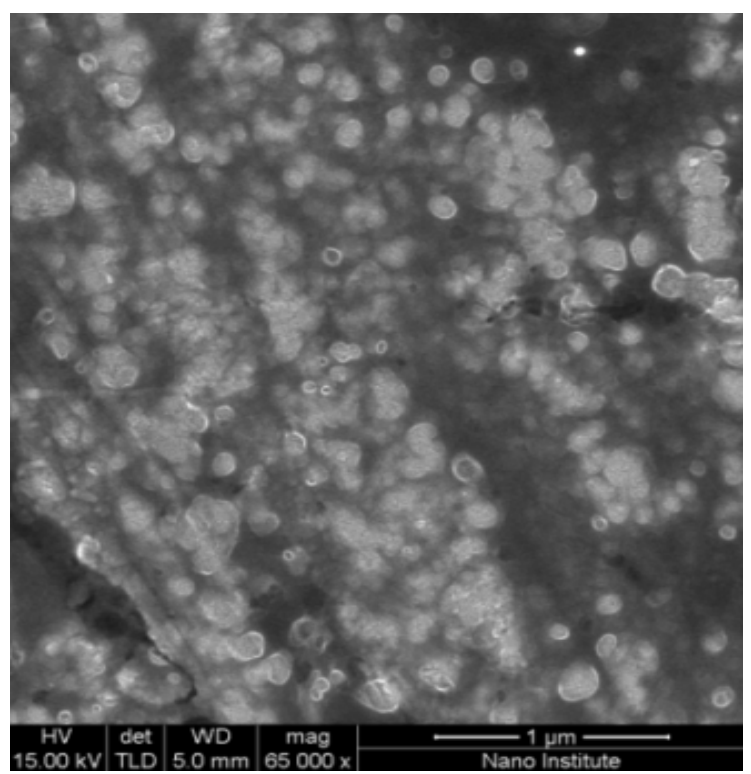

0 day of storage

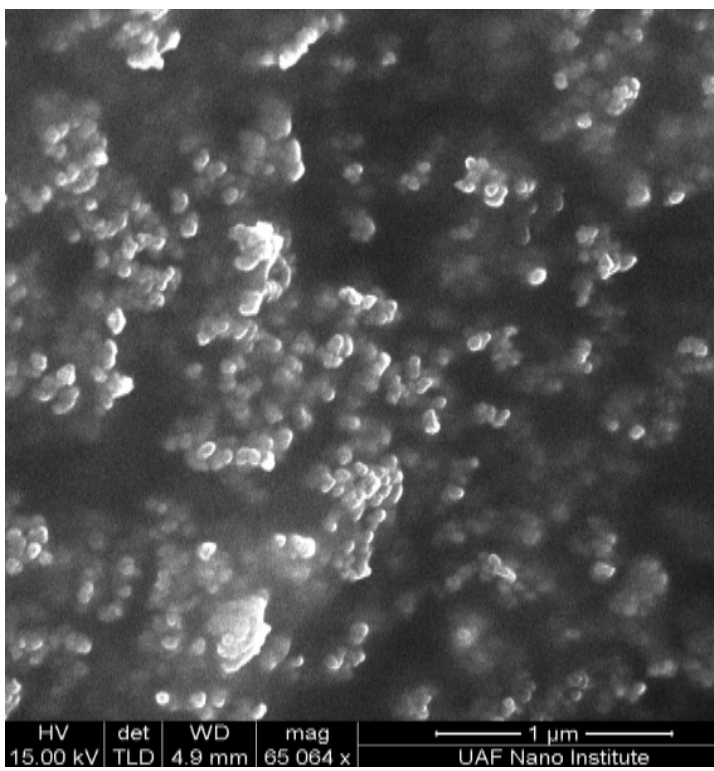

60 days of storage

Figure 6: SEM image of nano-encapsulated pentapeptide in apple juice. Images were taken at 0 and 60 days of storage. Magnification image is $65,000 X$. Diameter of particle size: 82 to $83 \mathrm{~nm}$.

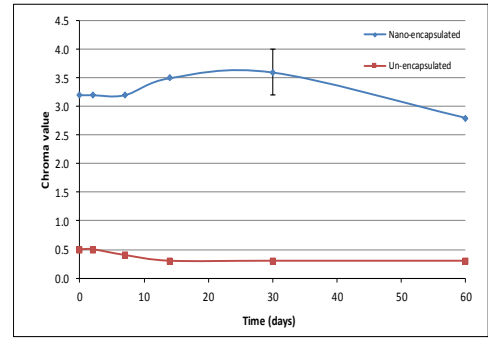

The Chroma value changes

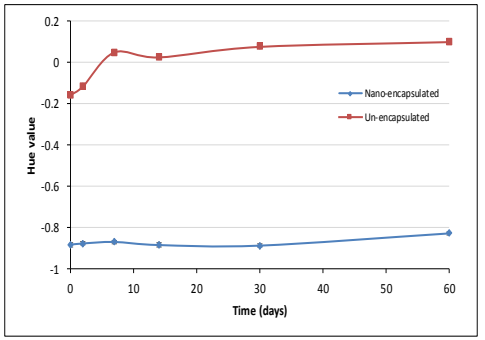

The Hue value changes

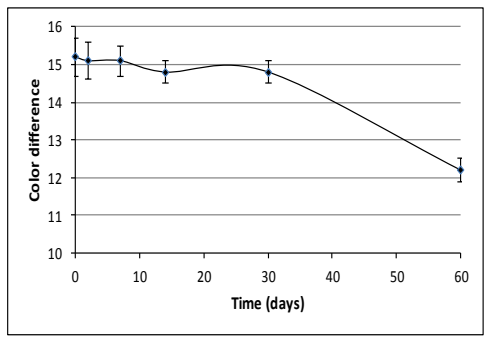

The color different chances

Figure 7: The Chroma and Hue values of nano-encapsulated and un-encapsulated pentapeptides incorporated in apple juice, and color differences between nano-encapsulated and un-encapsulated over a storage period $(0,2,7,14,30$, and 60 days). The values are represented as means of replicate analysis \pm standard deviation with $P$ value $<0.001$.

of these bioactives can potentially maintain the stability and prevent degration of bioactive peptides during storage period [22].

\section{Acknowledgement}

The authors would like to acknowledge the contributions of Dr. Kevin Thompson (Agricultural Statistics) and Dr. Suresh Kumar Thallapuranam (Department of Chemistry) in the University of Arkansas for their advice and support in conducting this research.

\section{References}

1. Kannan A, Hettiarachchy N, Narayan S (2009) Colon and breast anti-cancer effects of peptide hydrolysates derived from rice bran. Open Bioactive Comp J 2: 17-20.

2. Kannan A, Hettiarachchy N, Johnson MG, Nannapaneni R (2008) Human colon and liver cancer cell proliferation inhibition by peptide hydrolysates derived from heat-stabilized defatted rice bran. J Agric Food Chem 56: 11643-11647.

3. Kleiman S, Ng SW, Popkin B (2012) Drinking to our health: can beverage companies cut calories while maintaining profits? Obes Rev 13: 258-274.
4. Khairallah $G$ (2011) Stability and sensory properties of rice bran peptide fraction incorporated orange juice. [MS Thesis] University of Arkansas: Fayetteville, AR. Available from: University library.

5. Acosta E (2009) Bioavailability of nanoparticles in nutrient and nutraceutical delivery. Curr Opinion Coll Interf Sci 14: 3-15.

6. Zimet P, Rosenberg D, Livney, YD (2011) Re-assembled casein micelles and casein nanoparticles as nano-vehicles for ?-3 polyunsaturated fatty acids. Food Hydroc 25: 1270-1276.

7. García-Nebot MJ, Alegría A, Barberá R, Clemente G, Romero F (2009) Does the addition of caseinophosphopeptides or milk improve zinc in vitro bioavailability in fruit beverages? Food Res Internl 42: 1475-1482.

8. Ravichandran M, Hettiarachchy NS, Ganesh V, Ricke SC, Singh S (2011) Enhancement of antimicrobial activities of naturally occurring phenolic compounds by nanoscale delivery against Listeria monocytogenes, Escherichia coli O157: $\mathrm{H} 7$ and Salmonella typhimurium in broth and chicken meat system. J Food Safety 31: 462-471.

9. Korhonen H, Pihlanto A (2003) Food-derived bioactive peptides--opportunities for designing future foods. Curr Pharm Des 9: 1297-1308. 
Citation: Alessa F, Hettiarachchy N, Rayaprolu SJ, Benamara M, Greathouse D, et al. (2014) Stability of Nano Encapsulated Rice Bran Derived Bioactive Pentapeptide in Apple Juice. J Food Process Technol 5: 356. doi:10.4172/2157-7110.1000356

10. Utsunomiya S, Ewing RC (2003) Application of high-angle annular dark field scanning transmission electron microscopy, scanning transmission electron microscopy-energy dispersive X-ray spectrometry, and energy-filtered transmission electron microscopy to the characterization of nanoparticles in the environment. Environ Sci Technol 37: 786-791.

11. Musumeci T, Ventura CA, Giannone I, Ruozi B, Montenegro L, et al. (2006) PLA/PLGA nanoparticles for sustained release of docetaxel. Int J Pharm 325: $172-179$

12. Hafner B (2007) Scanning electron microscopy primer, characterization facility. University of Minnesota-Twin Cities

13. Evrendilek GA, Jin ZT, Ruhlman KT, Qiu X, Zhang QH, et al. (2000) Microbial safety and shelf-life of apple juice and cider processed by bench and pilot scale PEF systems. Innov Food Sci Emerging Technol 1: 77-86.

14. Toll H, Oberacher H, Swart R, Huber CG (2005) Separation, detection, and identification of peptides by ion-pair reversed-phase high-performance liquid chromatography-electrospray ionization mass spectrometry at high and low $\mathrm{pH}$. J Chrom A 1079: 274-286.

15. Tkachenko AG, Xie H, Coleman D, Glomm W, Ryan J, et al. (2003) Multifunctional gold nanoparticle-peptide complexes for nuclear targeting. J Am Chem Soc 125: 4700-4701
16. Selen Burdurlu H, Karadeniz F (2003) Effect of storage on nonenzymatic browning of apple juice concentrates. Food Chem 80: 91-97.

17. Kostanski JW, Thanoo BC, DeLuca PP (2000) Preparation, characterization and in vitro evaluation of 1- and 4-month controlled release orntide PLA and PLGA microspheres. Pharm Dev Technol 5: 585-596.

18. Gerhauser C (2008) Cancer chemopreventive potential of apples, apple juice and apple components. Planta Med 74: 1608-1624.

19. Alberto MR, Rinsdahl Canavosio MA, Manca de Nadra MC (2006) Antimicrobial effect of polyphenols from apple skins on human bacterial pathogens. Electr J Biotechn 9: 205-209.

20. Makadia HK, Siegel SJ (2011) Poly Lactic-co-Glycolic Acid (PLGA) as Biodegradable Controlled Drug Delivery Carrier. Polymers (Basel) 3: 13771397.

21. Schubert S, Delaney Jr JT, Schubert US (2011) Nanoprecipitation and nanoformulation of polymers: from history to powerful possibilities beyond poly (lactic acid). Soft Matter 7: 1581-1588.

22. Delehanty JB, Boeneman K, Bradburne CE, Robertson, K, Bongard JE (2010) Peptides for specific intracellular delivery and targeting of nanoparticles: implications for developing nanoparticle-mediated drug delivery. Therapeutic Delivery 1: 411-433. 